\title{
Reprovações e Trancamentos nas Disciplinas de Introdução à Programação da Universidade de São Paulo: Um Estudo Preliminar
}

\author{
Yorah Bosse $^{1}$, Marco Aurélio Gerosa ${ }^{1}$ \\ ${ }^{1}$ Instituto de Matemática e Estatística - Departamento de Ciência da Computação - \\ Universidade de São Paulo (USP) \\ Rua do Matão, 1010 - CEP 05508-090 - São Paulo - SP - Brasil \\ \{yorah,gerosa\}@ime.usp.br
}

\begin{abstract}
The Introduction to Programming course introduces programming logic to students, fostering creative and systematic thinking. However, many authors claim it has high failure rates. With the intent of investigating this statement, we have collected results from the University of São Paulo courses from 2010 to 2014. The failure rate was about 30\%, and in many cases superior to 50\%. These results have shown to be constant throughout the years, with more than $25 \%$ of the failing students attending the course twice or more. Compared to the other courses of the same semester of the bachelor course, Introduction to Programming has one of the highest failure rate.
\end{abstract}

Resumo. A disciplina de introdução à programação ensina aos alunos a lógica da programação de computadores, desenvolvendo o pensamento sistemático e criativo. Entretanto, muitos autores a apontam como sendo uma das disciplinas que os alunos mais reprovam. Com o intuito de investigar tal afirmação, realizamos um levantamento dos resultados das disciplinas de introdução à programação na USP de 2010 a 2014. O índice de reprovação na disciplina foi de 30\%, sendo que em vários casos ultrapassam os 50\%. Esses dados mantemse constantes ao longo dos anos e mais de $25 \%$ dos alunos aprovados neste período fizeram 2 ou mais vezes a disciplina. Comparando-se com disciplinas do mesmo semestre, nos cursos de computação, ela é uma das que mais reprova.

\section{Introdução}

Introdução à programação é uma disciplina que constitui a grade curricular de diversos cursos das áreas de exatas, humanas e biológicas. Muitos são os nomes atribuídos a ela, como Introdução à Programação, Introdução à Ciência da Computação, Introdução à Programação de Computadores, Lógica de Programação, Algoritmos, entre outras. Neste artigo será utilizado o nome Introdução à Programação (IP).

A disciplina de IP tem como principal objetivo fazer com que o aluno saiba desenvolver algoritmos, que, segundo Forbellone e Eberspächer [2005], pode ser definido como uma sequência de passos ordenados que visam atingir um objetivo específico. As disciplinas de IP adotam linguagens de programação como C, C++, Java, Python, VBA, entre outras, para treinar a aplicar os conceitos passados em sala de aula.

Porém, os alunos sentem uma grande dificuldade em entender estes conceitos, levando a reprovações ou trancamentos na disciplina e até desistência do curso [Santos e Costa 2006]. Poucos trabalhos acadêmicos quantificam esses percentuais, tanto em 
termos temporais quanto relativos a outras disciplinas cursadas pelos alunos. Gestores, professores e desenvolvedores de ferramentas educacionais possuem poucos parâmetros para guiar suas ações no sentido de melhorar a qualidade do ensino de programação. Os alunos também ficam sem base para comparar seu desempenho com os demais.

Esta pesquisa foi realizada com o principal objetivo de investigar o percentual de reprovações na Universidade de São Paulo (USP) com o intuito de se obter dados para embasar pesquisas subsequentes e se obter um melhor entendimento da realidade atual. A USP oferece a disciplina para 25 cursos de graduação diferentes em diferentes campi no estado de São Paulo. Foram levantados dados de 5 anos e, no total, foram consideradas 19.859 matrículas individuais de alunos. As questões de pesquisa que este estudo investigou estão listadas na Tabela 1.

\section{Tabela 1: Questões de pesquisa}

\begin{tabular}{l|l}
\hline \multicolumn{1}{c}{ Questões de Pesquisa } \\
\hline QP1 & $\begin{array}{l}\text { Qual o percentual de reprovações / trancamentos das disciplinas de } \\
\text { introdução à programação da USP? }\end{array}$ \\
\hline QP2 & Qual é a evolução temporal deste percentual? \\
\hline QP3 & $\begin{array}{l}\text { Como se mostra tal percentual analisando-se isoladamente as diversas } \\
\text { disciplinas de introdução à programação da USP? }\end{array}$ \\
\hline QP4 & Como se compara este percentual com outra disciplina do mesmo semestre? \\
\hline QP5 & $\begin{array}{l}\text { Quantos vezes o aluno frequenta a disciplina de introdução à programação } \\
\text { antes de obter aprovação? }\end{array}$ \\
\hline
\end{tabular}

Este artigo está organização em 7 Seções, sendo esta a primeira. Na Seção 2, temos a revisão da literatura. $\mathrm{Na}$ Seção 3 é apresentada a metodologia do estudo, seguida dos resultados na Seção 4. Na Seção 5 discorremos sobre as limitações e ameaças à validade do estudo. Na Seção 6 apresentamos a conclusão e, em seguida, na Seção 7, as referências bibliográficas.

\section{Revisão da Literatura}

Entender e aplicar os conceitos estudados nas disciplinas de introdução à programação é fundamental para que o aluno consiga se desenvolver em programações de sistemas mais avançados. Helminen e Malmi [2010] afirmam que a programação é uma competência essencial nos cursos de Ciência da Computação. Porém, normalmente os estudantes apresentam uma grande dificuldade em assimilar os conteúdos abordados, ocasionando em reprovação ou desistência. Corney e colegas [2010] afirmam que em média 30\% dos alunos da disciplina de IP da Universidade de Queensland Tecnology não conseguiram ser aprovados.

É muito comum ouvirmos os estudantes comentarem da dificuldade que possuem na disciplina de IP. Piteira e Costa [2013] afirmam que a programação pode ser um processo doloroso para os programadores iniciantes, pois eles precisam ter conhecimento declarativo e procedural, memorização, compreensão, resolução de problemas, abstração e capacidade de raciocínio lógico, entre outros. Os professores desta disciplina, segundo Jantan e Aljunid [2012], afirmam que a maior dificuldade encontrada pelos alunos é a compreensão de estruturas de programação.

Os alunos apresentam muitas dificuldades para entender a lógica de programação, que requer uma prática intensiva para ser desenvolvida. Nas turmas geralmente existe uma quantidade muito grande de alunos, o que impossibilita o professor de acompanhar 
individualmente cada um. Além disso, Araújo (2007) afirma que "é muito comum, nas etapas iniciais de uma disciplina de programação, deparar-se com uma dificuldade em conseguir identificar o problema a ser resolvido e isso, seguramente, impede o desenvolvimento do raciocínio e, consequentemente, o aprendizado da programação".

Uma pesquisa feita por Campos [2010] em quatro instituições de ensino superior (IES) do Distrito Federal (DF) constatou que mais de 60\% dos alunos eram reprovados na disciplina de IP a cada semestre. Com dados de uma pesquisa realizada por Bennedsen e Caspersen [2007], com a participação de 63 instituições de diversos países, obtiveram um índice de $67 \%$ de aprovações.

A dificuldade na disciplina de IP pode estar entre os motivos que fazem com que os cursos da área de computação estejam entre os maiores no índice de evasões. Uma pesquisa realizada por Silva Filho e colegas [2007] mostra que a média de evasão nos cursos superiores no Brasil, entre os anos 2000 e 2005, era de $22 \%$ e, os cursos da área de computação estavam entre os maiores, chegando à $36 \%$.

\section{Metodologia}

Neste estudo, foi feito um levantamento, que segundo [Creswell 2010] apresenta uma descrição quantitativa dos dados de uma determinada população. A população do presente estudo são os alunos das disciplinas de IP da Universidade de São Paulo de 2010 a 2014.

A primeira etapa para obter os dados para análise, foi escolher as disciplinas que iriam compor o estudo. As disciplinas foram selecionadas por meio de uma pesquisa no sistema acadêmico da universidade, chamado de Sistema Jupiter ${ }^{1}$, com as seguintes palavras chaves: "programação", "algoritmos" e "computação". A Tabela 2 apresentada a relação da quantidade apresentada pelo sistema e a quantidade escolhida para a análise.

Tabela 2: Totais de disciplinas apresentadas pelo Sistema Jupiter para cada palavra de busca utilizada

\begin{tabular}{l|c|c}
\hline \multicolumn{1}{c|}{ Palavra de Busca } & $\begin{array}{c}\text { Total de } \\
\text { Disciplinas } \\
\text { Apresentadas }\end{array}$ & $\begin{array}{c}\text { Total de } \\
\text { Disciplinas } \\
\text { Escolhidas }\end{array}$ \\
\hline Programação & 66 & 12 \\
\hline Algoritmos & 29 & 0 \\
\hline Computação & 112 & 19 \\
\hline Total & 207 & 31 \\
\hline
\end{tabular}

Para cada disciplina apresentada, foi feita uma seleção manual, onde o plano de ensino de cada uma foi obtido e seu programa resumido e completo analisados. Os critérios de inclusão e exclusão estão listados na Tabela 3.

\footnotetext{
${ }^{1}$ Disponível no site: https://uspdigital.usp.br/jupiterweb/jupDisciplinaBusca?tipo=D\&codmnu=4526
} 
Tabela 3: Critérios de inclusão e exclusão das disciplinas apresentadas pelo Sistema Jupiter da USP.

\begin{tabular}{|c|c|c|c|}
\hline \multicolumn{2}{|r|}{ Critérios de Inclusão } & \multicolumn{2}{|r|}{ Critérios de Exclusão } \\
\hline CI1 & $\begin{array}{l}\text { Ter no seu programa pelo menos } 4 \text { dos } \\
\text { seguintes assuntos: } \\
\Rightarrow \quad \text { Conceitos de linguagens de } \\
\text { programação, por exemplo: tipos de } \\
\text { variáveis } \\
\Rightarrow \quad \text { Estruturas de programação: } \\
\text { seleção e repetição } \\
\Rightarrow \quad \text { Variáveis estruturadas ou tipos de } \\
\text { dados compostos: vetores e matrizes } \\
\Rightarrow \quad \text { Modularização de programas: } \\
\text { funções e/ou procedimentos } \\
\Rightarrow \quad \text { Utilização de alguma linguagem } \\
\text { de programação }\end{array}$ & CE1 & $\begin{array}{l}\text { Tratar de uma comparação de } \\
\text { paradigmas de programação como } \\
\text { programação funcional, procedural, } \\
\text { orientada à objetos, entre outras. Sendo } \\
\text { feita programação para ver o } \\
\text { funcionamento de cada uma. }\end{array}$ \\
\hline
\end{tabular}

Das 31 disciplinas selecionadas, apenas 29 foram utilizadas neste estudo, pois as disciplinas de código SCC0222 e SCC0221 foram ativadas apenas no ano de 2015, logo não possuem dados dentro do período estipulado por esta pesquisa.

Os dados para análise foram disponibilizados pela USP, após autorização da PróReitoria de Graduação, com os campos que identificam os alunos alterados para números aleatórios mas consistentes, para que pudéssemos identificar unicamente o aluno dentro da análise.

\section{Resultados}

O banco de dados utilizado na pesquisa possui para cada disciplina as seguintes informações: cursos que a contém na grade curricular, históricos dos alunos que se matricularam nela no período de 2010 e 2014 e os dados referentes às turmas abertas, contendo ano, semestre, professor ministrante, identificador do aluno, frequência, nota e situação, entre outras informações.

Nos cinco anos analisados, ocorreram 20.948 matrículas em todas disciplinas juntas, das quais consideramos 19.853. As 1.095 matrículas desconsideradas são as que correspondem à situação "Não cadastrado", não tendo como identificar se nesta matrícula o aluno foi ou não aprovado. Em primeira análise, verificamos que dessas 19.853 matrículas, $70,69 \%$ resultaram em aprovação, $26,70 \%$ em reprovação por nota e/ou frequência e 2,61 em trancamentos. Na USP, em uma escala de 0 a 10, a média de aprovação direta é de 5,0 e para fazer a recuperação é de 3,0. Nesta análise foram considerados todos alunos aprovados, antes e depois da recuperação. Os dados desta análise estão representados na Figura 1A. 


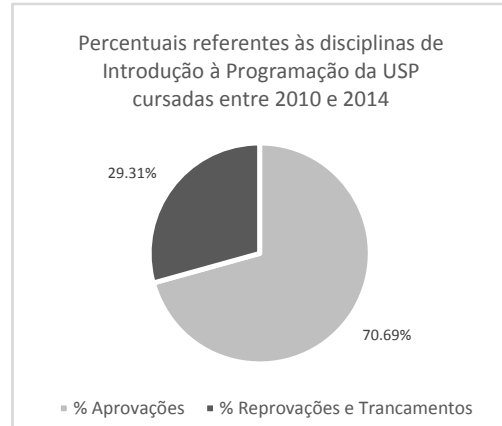

(A)

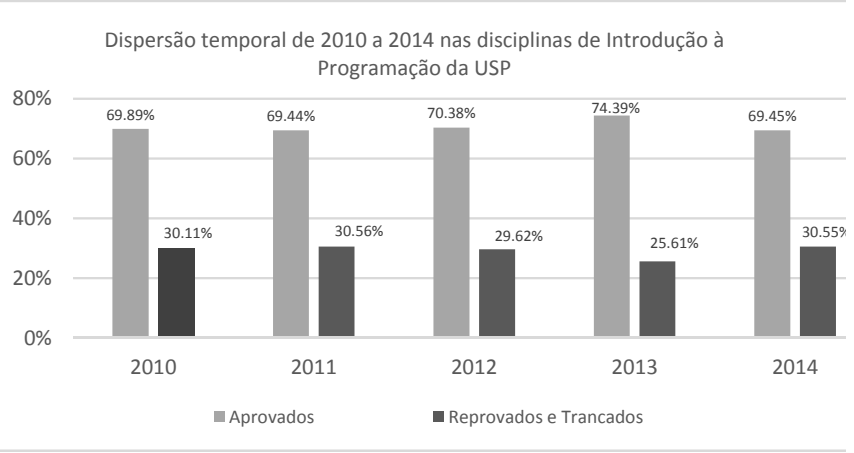

(B)

Figura 1. (A) Percentual29, geral e (B) Dispersão temporal das aprovações e reprovações / trancamentos nas disciplinas de IP na USP, no período de 2010 a 2014.

A dispersão temporal destes dados está representada na Figura 2. Estatisticamente, não é possível afirmar que há diferença significativa entre os anos analisados quanto às médias de reprovação, segundo o teste não-paramétrico de Kruskal-Wallis, no qual foi obtido valor $\mathrm{p}$ de 0,9829 .

Analisando este percentual em cada disciplina (Figura 2), obtivemos um resultado bastante variado, sendo que a disciplina que mais reprovou chegou a $62,18 \%$ de reprovações/trancamentos. Em contrapartida, a que menos reprovou teve 5,91\% de reprovações/trancamentos. São vários os fatos que podem levar à esta diferença de resultados, como perfil dos alunos, método de ensino aplicado ou até mesmo o nível de dificuldade que a disciplina foi aplicada e cobrada. As turmas têm em média 55 alunos e não encontramos correlação estatística nem entre o tamanho da turma e a nota média e nem entre o tamanho da turma e o percentual de reprovados. Neste artigo não faremos um estudo mais aprofundado desses fatores.

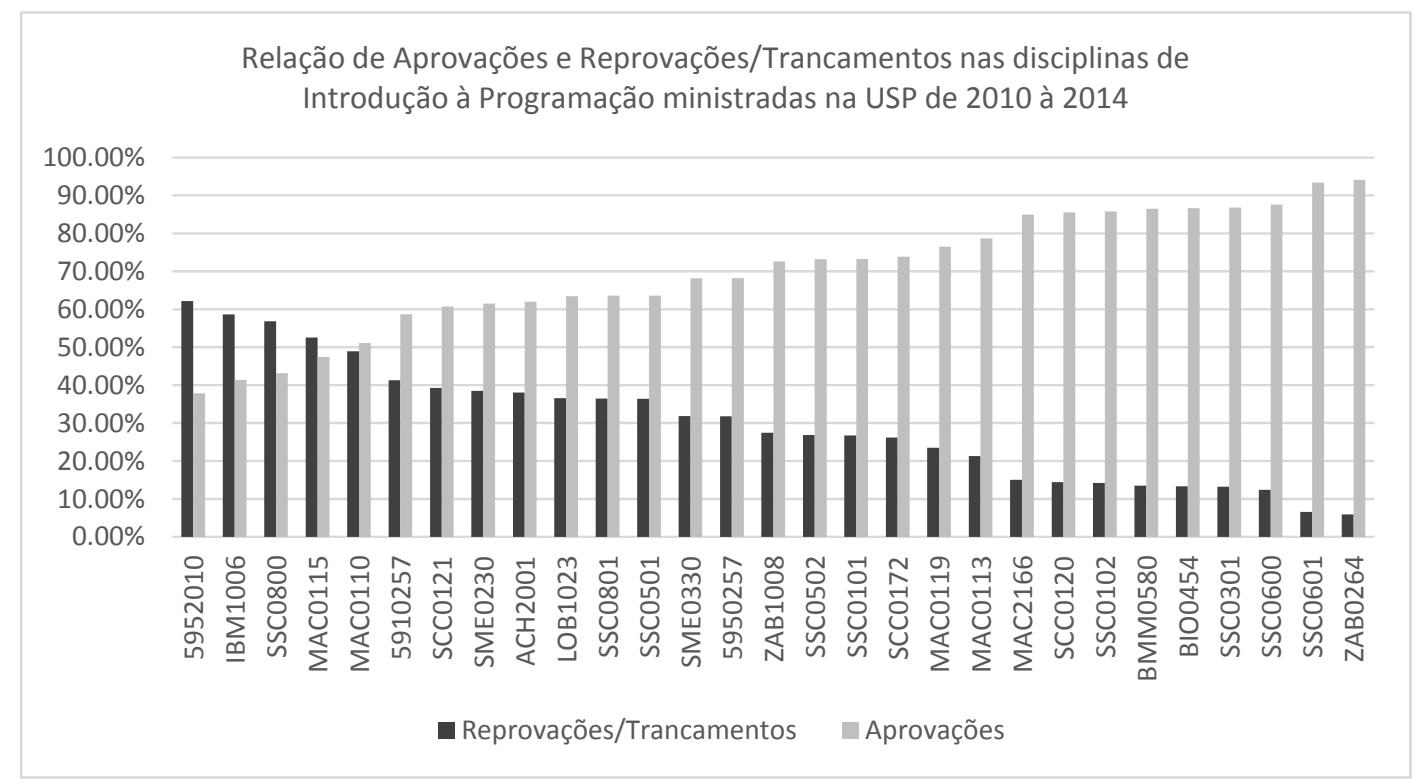

Figura 2. Percentual de aprovações e reprovações / trancamentos, por disciplina de IP na USP, no período de 2010 a 2014. 
Outra análise realizada foi a quantidade de vezes que um aluno precisa cursar a disciplina antes de obter aprovação. Para esta análise foram considerados apenas os alunos que já possuíam aprovação no seu histórico escolar. Há aluno, por exemplo, que já se matriculou mais de 8 vezes na disciplina, sem ter obtido ainda a aprovação, e por isso não foi incluído na análise. A Figura 3 mostra o percentual de vezes que um aluno se matricula na disciplina de IP antes de obter aprovação.

\section{Quantidade de vezes que o aluno cursa a disciplina para obter aprovação}

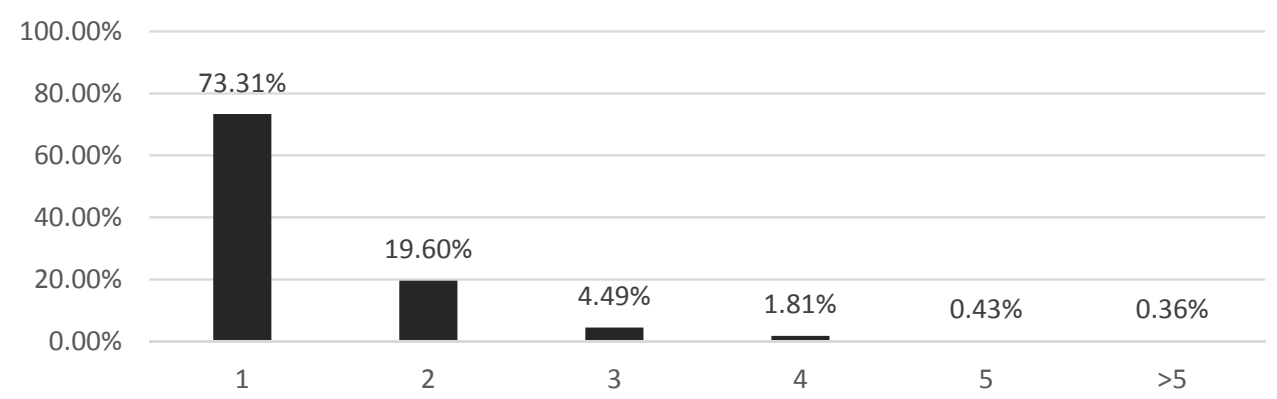

Figura 3. Percentual de vezes que alunos teve que cursar a disciplina de IP antes de obter aprovação

Pegando apenas alunos dos cursos de computação da USP (Ciência da Computação no IME e no ICMC, Sistemas de Informação na EACH e no ICMC e Engenharia de Computação na POLI e no ICMC) obtivemos os percentuais apresentados na Figura 4. Desconsideramos as disciplinas exclusivamente de laboratório.

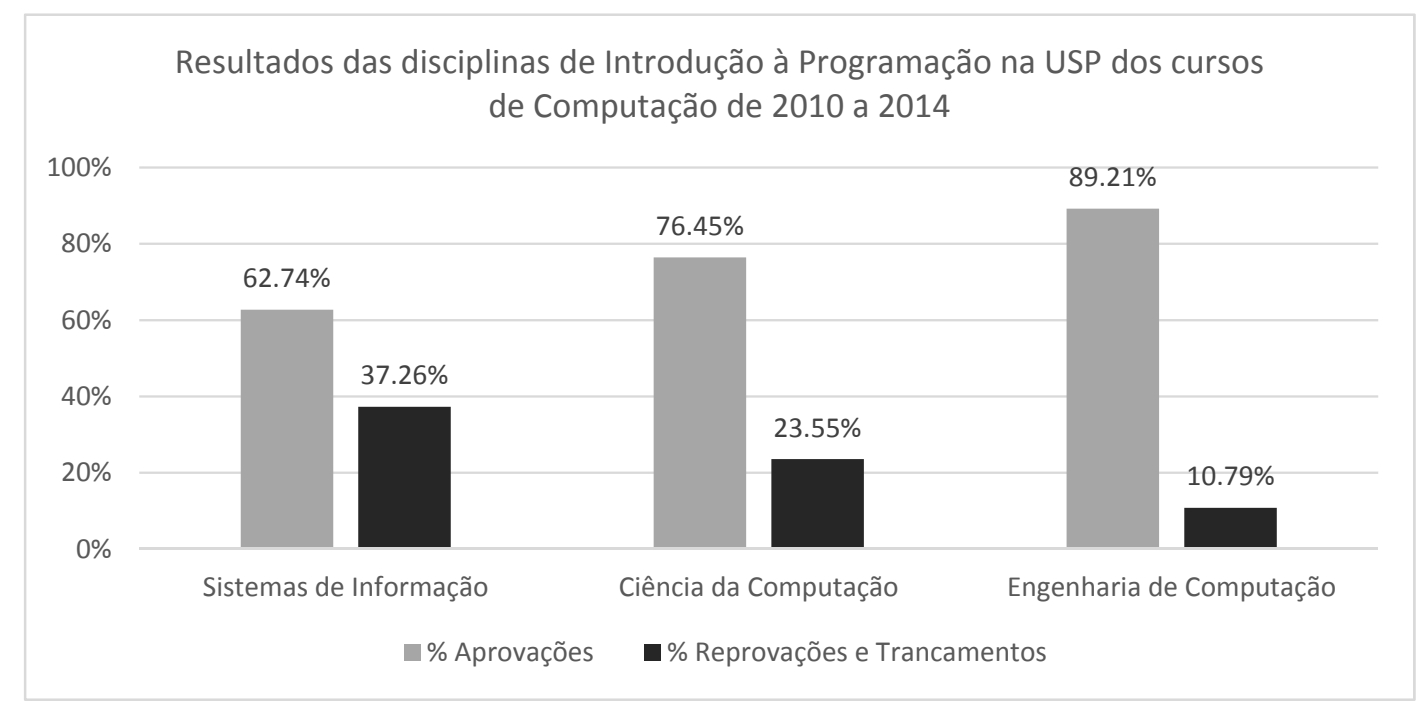

Figura 4. Percentual de aprovações e reprovações/trancamentos nas disciplinas de IP na USP, de 2010 a 2014, por curso da área de computação.

Para fazer uma análise comparativa com outra disciplina considerada de elevada dificuldade pelos alunos, buscamos casos em que o aluno cursou pela primeira vez uma disciplina de IP (MAC110, MAC2166 e ACH2001) no mesmo ano e semestre que cursou uma de cálculo (MAT0111, MAT2453, ACH2011), também pela primeira vez, no campus da capital. Os dados mostram que existe uma relação bastante próxima nas 
médias e medianas das duas disciplinas. Uma vez analisados o gráfico de normalidade dos dados e os valores p obtidos pelo teste de normalidade Shapiro-Wilk com um nível de significância de 5\%, concluímos que não há evidência para afirmar que a amostra não foi retirada de uma população normalmente distribuída. Comparando as disciplinas de Cálculo e Introdução à Computação, não há indício para concluir, com $99 \%$ de confiança, que a média para ambas disciplinas são diferentes.

Tabela 4: Relação de Sigla, média, mediana e desvio padrão de uma disciplina de IP e uma de cálculo, cursadas pela primeira vez no mesmo semestre do mesmo ano, nos cursos da área de computação na USP.

\begin{tabular}{|l|c|c|c|c|c|c|c|c|}
\hline & \multicolumn{4}{|c|}{ Introdução à Programação } & \multicolumn{4}{c|}{ Cálculo } \\
\cline { 2 - 10 } & Sigla da Disciplina & Média & Mediana & Desvio Padrão & Sigla da Disciplina & Média & Mediana & Desvio Padrão \\
\hline Ciência da Computação & MAC0110 & 5,41 & 6,80 & 3,50 & MAT0111 & 5,57 & 5,80 & 2,86 \\
\hline Engenharia de Computação & MAC2166 & 6,71 & 7,10 & 2,16 & MAT2453 & 5,68 & 5,70 & 1,80 \\
\hline Sistemas de Informação & ACH2001 & 1,46 & 0,50 & 1,97 & ACH2011 & 2,11 & 1,00 & 2,53 \\
\hline
\end{tabular}

Além disto, foram computados o total de aprovações e reprovações existentes, nas disciplinas de primeiro semestre das grades curriculares dos cursos de computação dos campi da capital (Figura 5). Cabe ressaltar que algumas disciplinas fazem parte da grade curricular de vários cursos fora da área de computação. Podemos observar que IP é uma das que mais reprovam.

\section{Limitações e Ameaças à Validade}

Alguns fatores podem levar a possibilidade de erros nos dados divulgados, como a possiblidade de erro na extração e compilação dos dados do sistema. Para evitar isso, foram selecionados vários dados para checagem manual, comparando com outras fontes (planilha de professor, relatório do sistema, etc.).

$\mathrm{Na}$ separação por cursos da área de informática, o fato de, em alguns anos, os alunos da Escola Politécnica de Engenharia optarem após o ciclo básico pela habilitação em Engenharia da Computação pode fazer com que seus dados não tenham sido contabilizados. Além disso, a codificação do curso de Engenharia de Computação apresentou mudanças, que podem ter feito a não contabilização dos dados de alguns dos anos.

Outro fator relevante é o das matrículas que possuem situação final "Não Cadastrado". Esta situação refere-se ao fato da disciplina estar vinculada a uma outra e, nesta estar a situação final. Estes casos foram desconsiderados neste estudo e isto poderia gerar alguma diferença quanto ao percentual de aprovações e reprovações/trancamentos. 


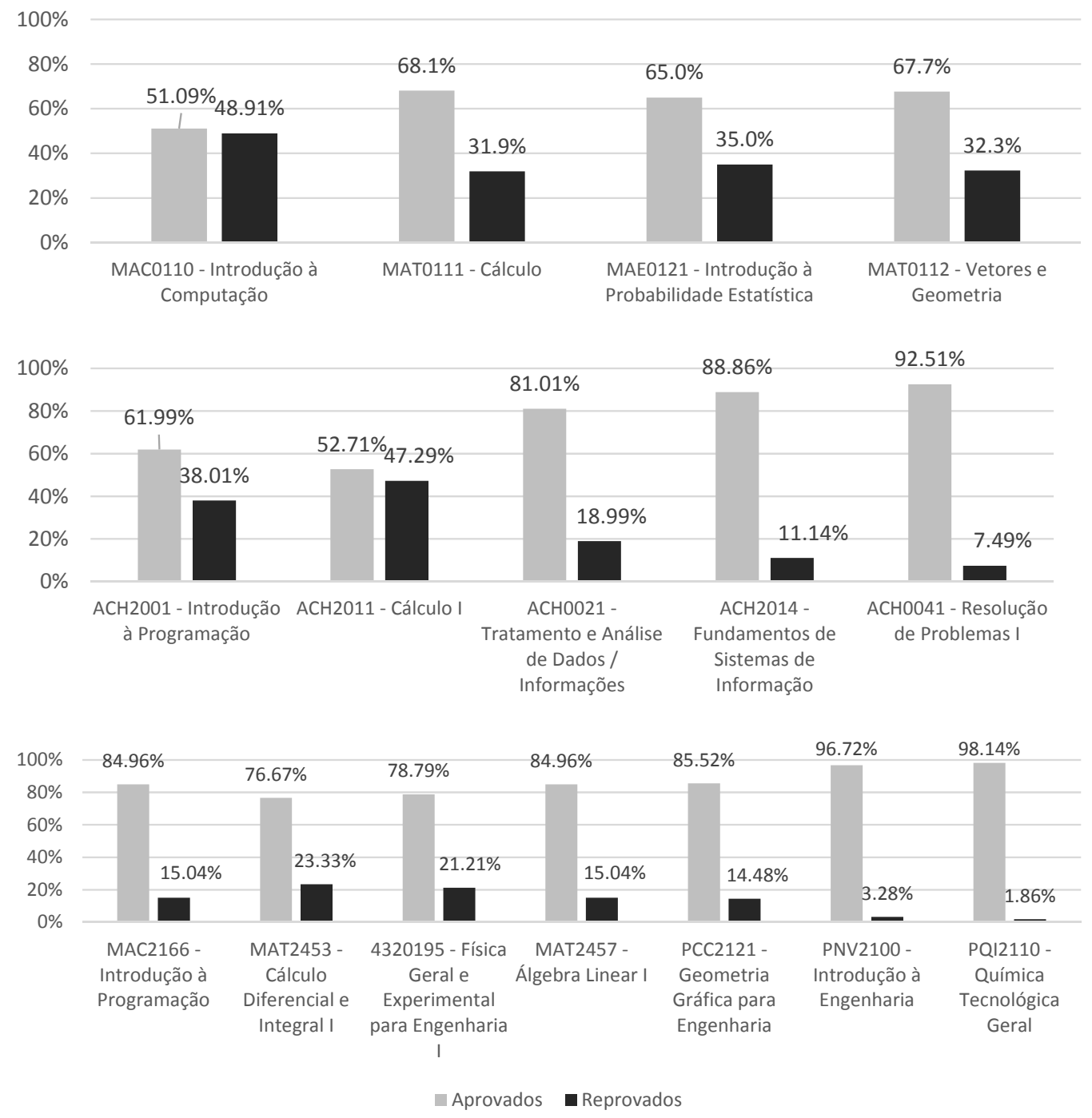

Figura 5. Percentual de aprovações e reprovações/trancamentos por disciplina de primeiro semestre de cursos da área de computação dos campi de São Paulo capital

\section{Conclusão}

Os resultados apresentados pelas análises dos bancos de dados disponibilizados pela USP corroboraram com os resultados de outros estudos [Bennedsen e Caspersen 2007]. Considerando todas as disciplinas de IP lecionadas na USP, o percentual médio de reprovações / trancamentos é de $29,31 \%$ e, no decorrer dos cinco anos, este percentual manteve-se praticamente constante.

Analisando-se as disciplinas individualmente, este percentual mostrou-se bastante variado, indo de $62,18 \%$ a $5,91 \%$ de reprovações. Outro dado que foi levantado é que mais de $25 \%$ dos alunos que foram aprovados no período de 2010 a 2014 tiveram que cursar 2 ou mais vezes a disciplina de IP e que, essa disciplina é uma das que mais reprova entre as do primeiro semestre dos cursos de computação. 
Sugestão de trabalho futuro é fazer uma análise separada para cursos de graduação em computação e graduação em outras áreas. Os resultados analisando-se apenas os cursos da área de computação poderiam trazer alguma novidade relevante para futuras pesquisas e também como informações para auxiliar coordenadores, professores e alunos. Outra sugestão é a investigação dos motivos que levam a existir uma variação tão elevada de reprovações entre as disciplinas de IP, buscando descobrir se é a metodologia aplicada ou o nível de cobrança ou se alguma ferramenta de ensino aprendizagem é utilizada. Podem ser feitas também análises das notas obtidas nas disciplinas em comparação com a nota de corte para entrar nos cursos.

\section{Agradecimentos}

Gostaria de agradecer à Edmeia Ferreira Martins, Chefe Administrativo do Serviço de Apoio à Gestão Acadêmica, por ter providenciado que nosso pedido de dados fosse autorizado. Ao Daniel G. Konno, do Serviço de Apoio à Gestão dos Sistemas de Informação, pela organização e formatação dos dados utilizados. À Virginia Carrara pelo desenvolvimento da parte estatística do artigo.

\section{Referências}

Araújo, E. (2007). Algoritmos: Fundamento e Prática. $3^{\mathrm{a}}$ ed. ampl. e atual. Florianópolis: VisualBooks.

Barone, P. (2012). Diretrizes Curriculares Nacionais para os cursos de graduação em Computação. Disponível no site: http://portal.mec.gov.br/index.php? option $=$ com_docman\&task=doc_download\&gid= 8128\&Itemid=, em 04/04/2015.

Bennedsen, J. e Caspersen, M. (2007). Failure Rates in Introductory Programming. The SIGCSE Bulletin, Vol.39. N.2. June.

Campos, R. (2010). Metodologia ERM2C: Para melhoria do processo de ensinoaprendizagem de lógica de programação. XXX Congresso da Sociedade Brasileira de Computação - CSBC. XVIII Workshop sobre Educação em Computação - WEI. Belo Horizonte $-\mathrm{MG}$.

Corney, M., Teague, D., Thomas, R. (2010). Engaging students in programming. Em: ACE '10 Proceedings of the Twelfth Australasian Conference on Computing Education - Volume 103, pp. 63-72. Australia.

Creswell, J. (2010). Projeto de Pesquisa: métodos qualitativos, quantitativos e misto. $3^{\mathrm{a}}$. edição. Porto Alegre : Artmed.

Forbellone, A. e Eberspächer, H. (2005). Lógica de Programação : a construção de algoritmos e estruturas de dados. 3. ed. São Paulo : Pearson Prentice Hall.

Helminen, J. e Malmi, L. (2010). Jype - a program visualization and programming exercise tool for python. Em: SOFTVIS '10 Proceedings of the 5th international symposium on Software visualization. pp.153-162. USA.

Jantan, S., Aljunid, S. (2012). An experimental evaluation of scaffolded educational games design for programming. Em: ICOS 2012 - IEEE Conference on Open Systems.

Paula, L., Piva Junior, D. e Freitas, R. (2009). A Leitura e a Abstração do Problema no Processo de Formação do Raciocínio Lógico-Abstrato em Alunos. XVII Workshop 
sobre Educação em Computação - XXIX Congresso da Sociedade Brasileira de Computação. Bento Gonçalves - RS.

Piteira, M., Costa, C.: Learning computer programming: Study of di_culties in learning programming (2013). Em: ISDOC'13 - Proceedings of the 2013 International Conference on Information Systems and Design of Communication. pp. 75-80. USA

Santos, R. e Costa, H. (2006). Análise de Metodologias e Ambientes de Ensino para Algoritmos, Estruturas de Dados e Programação aos Iniciantes em Computação e Informática. In: INFOCOMP, Volume 5, $\mathrm{n}^{\mathrm{o}}$.1, ISSN 1807-4545.

Silva Filho, R., Montejunas, P., Hipólito, O. e Lobo, M. (2007). A Evasão no ensino superior brasileiro. Caderno de Pesquisa, v. 37, n. 132, p. 641-659, set/dez. 2007. Disponível em: http://www.scielo.br/pdf/cp/v37n132/a0737132.pdf 